\title{
The combination of folate receptor-positive circulating tumor cells and serum tumor markers suggests a histological diagnosis of lung cancer
}

\author{
Xing Lv"$^{\#}$, Shuo Wu", Xi Xu, Lei Wu, Ning Chang, Jian Zhang, Xinyu Ti \\ Department of Pulmonary and Critical Care Medicine, Xijing Hospital, Fourth Military Medical University, Xi'an, China \\ Contributions: (I) Conception and design: J Zhang; (II) Administrative support: X Ti; (III) Provision of study materials or patients: L Wu, N Chang; \\ (IV) Collection and assembly of data: X Xu, X Lv, S Wu; (V) Data analysis and interpretation: X Lv, S Wu; (VI) Manuscript writing: All authors; (VII) \\ Final approval of manuscript: All authors. \\ "These authors contributed equally to this work. \\ Correspondence to: Xinyu Ti; Jian Zhang. Department of Pulmonary and Critical Care Medicine, Xijing Hospital, Fourth Military Medical University, \\ No. 169 Changle West Road, Xi'an 710032, China. Email: tixinyu@fmmu.edu.cn; 13991802890@163.com.
}

\begin{abstract}
Background: Folate-receptor alpha $(\mathrm{FR} \alpha)$ is overexpressed in lung carcinoma. The FR-positive circulating tumor cell (FR+ CTC) has been established to be a non-invasive biomarker for lung cancer diagnosis. In this study, we sought to examine the value of FR+ CTC in the histological diagnosis of suspicious spaceoccupying pulmonary lesions.

Methods: A total of 538 patients with suspicious space-occupying pulmonary lesions were enrolled in this study. FR+ CTCs were detected before treatment initiation using negative enrichment and ligand-targeted polymerase chain reaction assays. The enrolled patients concurrently received serum biomarker tests.

Results: A total of 282 lung cancer patients [163 with adenocarcinoma (ADC), 71 with squamous cell carcinoma (SCC), and 48 with small cell lung cancer (SCLC)], and 256 patients with benign disease who concurrently received FR+ CTC and serum biomarker tests were randomly assigned to a training set and a validation set. The FR+ CTC levels of patients with lung cancer were significantly higher than those of patients with benign disease $(\mathrm{P}<0.001)$. Compared to serum tumor biomarkers alone, the model combining FR+ CTC and serum biomarkers had the highest area under the receiver operating characteristic curve in the diagnosis of NSCLC, ADC, SCC, and SCLC.

Conclusions: Diagnostic models that include both FR+ CTC and serum biomarkers could increase the efficiency of distinguishing between different histological types of lung cancer and benign space-occupying pulmonary diseases.
\end{abstract}

Keywords: Biomarker; circulating tumor cell (CTC); folate receptor (FR); liquid biopsy; space-occupying pulmonary lesion

Submitted Nov 24, 2021. Accepted for publication Jan 14, 2022.

doi: $10.21037 /$ jtd-21-1975

View this article at: https://dx.doi.org/10.21037/jtd-21-1975

\section{Introduction}

Respiratory diseases are usually identified from clinical symptoms, such as coughing, hemoptysis, difficulty breathing, and chest pain. In clinical practice, the differential diagnosis between malignant and benign respiratory diseases is critical, as the treatment strategies are completely different (1). Benign respiratory diseases without spaceoccupying lesions, including bronchiectasis, pneumothorax, pneumonias, and chronic obstructive pulmonary disease (COPD), are easily identified by computed tomography (CT), X-ray, or a pulmonary function test. Often, CT or X-ray scans of patients with respiratory diseases also 
reveal pulmonary space-occupying lesions (2). However, the current routine methods for estimating the malignancy of space-occupying lesion suffer from technical problems; for example, radiological imaging modalities show interobserver disagreement (3), while tissue biopsy is not applicable to a significant portion of patients for a number of reasons, including the invasive nature of the biopsy process, the inaccessibility of the nodule due to its location, and an insufficient amount of tissue being obtained for a meaningful pathological evaluation (4).

Carcinoembryonic antigen (CEA), squamous cell carcinoma antigen (SCCA), neuron-specific enolase (NSE), and cytokeratin 19 fragments (Cyfra21-1) are widely used in the diagnosis of lung cancers. However, the utility of single biomarkers in the diagnosis of lung cancer is limited because they lack sufficient sensitivity and specificity, while at the same time, elevated levels of these biomarkers are often seen in patients with certain benign pulmonary diseases (5). The American Society of Clinical Oncology, the European Group on Tumor Markers (EGTM), and the National Academy of Clinical Biochemistry (NACB) recommend different combinations of these biomarkers to improve detection sensitivity and specificity $(6,7)$. The combination of CYFRA21-1, NSE, and CEA is recommended for the detection of lung cancer by the EGTM. The NACB recommends the measurement of four markers to identify the leading biomarker to suggest the most probable histology subtypes; NSE has high specificity for small cell lung cancer (SCLC); CEA has high specificity for adenocarcinoma (ADC); Cyfra21-1 has high specificity for squamous cell carcinoma (SCC) and which is the best marker for NSCLC, and SCCA has high specificity for SCC and provides additive information to Cyfra21-1. A number of multi-biomarker combination prediction models have been proposed and investigated in recent years $(8,9)$. However, no definitive combination model for malignancy prediction has been recommended.

Under a "liquid biopsy" approach, circulating tumor cells (CTCs) could be used to diagnose lung cancer (10). $\mathrm{FR}+\mathrm{CTC}$ test is based on the expression of folate-receptor alpha $(F R \alpha)$ in circulating cells of non-blood origin. FR $\alpha$ expression has been reported to be associated with the prognosis of lung cancers $(11,12)$. Thus, the expression of FR $\alpha$ in respiratory diseases could be a useful biomarker for guiding FR $\alpha$-based diagnostic and therapeutic strategies (13). The diagnostic efficiency of FR+ CTCs in NSCLC, lung adenocarcinoma small pulmonary nodules have been intensively studied (14-18), the aim of these studies usually to distinguish malignant lesions from healthy individuals or benign lung lesions that have not been specifically subdivided. In our study, we included cases of suspicious pulmonary space-occupying disease and subdivided patients with benign disease.

To improve diagnostic efficiency and prediction capacity, it is highly desirable that the appropriate combinations of biomarkers are selected for lung cancer diagnosis in clinical practice. Thus, we present the following article in accordance with the STARD reporting checklist (available at https://jtd.amegroups.com/article/view/10.21037/jtd-211975/rc) to explore the clinical significance of FR+ CTCs in combination with common serum biomarkers to improve the diagnostic efficiency of respiratory diseases with suspicious space-occupying lesions.

\section{Methods}

\section{Study design}

This prospective, single-center study was conducted at Xijing Hospital from December 2012 to July 2018. A total of 538 patients with suspicious pulmonary spaceoccupying lesions (e.g., granuloma, hamartoma, benign lung nodules, inflammatory pseudotumor, interstitial pulmonary disease, and tuberculosis), or malignant spaceoccupying lesions (e.g., ADC, SCC, and small cell lung cancer) were consecutive enrolled in the study. Patients with other subtypes of pulmonary diseases or without a definite diagnosis were excluded from the study.

Inclusion criteria: (I) 18-80 years old, (II) suspicious pulmonary space-occupying lesions $(\geq 8 \mathrm{~mm}$ by CT scanning), (III) preparation for surgery or biopsy for pathological confirmation and FR+ CTC testing completed prior to operation. Exclusion criteria: (I) patients with other severe diseases and not suitable for participate, (II) acute respiratory tract infection (fungal, bacterial, viral or tuberculosis) in the past one month prior to enrolment, (III) a history of lung cancer and received anti-tumour therapy, (IV) a history of other malignancies, single/ multiple pulmonary nodules are suspected to be metastatic carcinoma, (V) calcified nodules.

For the patients that underwent tissue biopsy, the diagnosis was based on a pathological evaluation of the diseased tissue by a professional pathologist. For the patients without clear pathological assessments, the diagnosis was made by a multidisciplinary team, which included members from the Department of Pulmonary Medicine, Oncology, 
Pathology, and Medical Imaging, according to the results of the thoracic CT scan, or laboratory examination.

The study was conducted in accordance with the Declaration of Helsinki (as revised in 2013). The ethics committee of the Xijing Hospital approved the study (No. KY20162056), and informed consent was obtained from each participant before study entry.

\section{FR+ CTC analysis}

A sample of 3 milliliters of peripheral blood was collected from each participant using an ethylenediaminetetraacetic acid (EDTA) anti-coagulant vacuum tube. Samples were stored at $4{ }^{\circ} \mathrm{C}$ and processed within 24 hours. FR+ CTC detection was performed using a Folate Receptorpositive Cell Detection Kit (Genosaber, Shanghai, China) as previously described (15). FR+ CTCs were first enriched using the negative enrichment method, in which erythrocytes were first lysed by a lysing buffer, and leukocytes were then depleted by a combination of anti-cluster of differentiation (CD) 45 and anti-CD 14 immunomagnetic beads. The enriched FR+ CTCs were then labelled by a FR $\alpha$-targeting probe that contained the conjugate of a folic acid and a synthesized oligonucleotide. The labelled FR+ CTCs were enumerated by quantitative polymerase chain reaction (PCR) using the proprietary ligand-targeted PCR method (17). A serial of standards containing oligonucleotides $\left(10^{-14}\right.$ to $10^{-9} \mathrm{M}$, corresponding to 2 to $2 \times 10^{5} \mathrm{FU} / 3 \mathrm{~mL}$ blood) were used for the FR+ CTC quantification. The folate-receptor unit $(\mathrm{FU})$ per $3 \mathrm{~mL}$ of peripheral blood was calculated from the standard curve and was used to determine the FR+ CTC level in each sample.

\section{Serum biomarker analysis}

A subgroup of patients concurrently received serum biomarker tests. An additional sample of $3 \mathrm{~mL}$ of coagulated blood was obtained from these patients. After centrifuging at $800-1,000 \mathrm{rpm}$ for 10 minutes, the serum was collected for serum biomarker tests, including CEA, CYFRA21-1, NSE, and SCCA, using the enzyme-linked immune-sorbent assay method (Roche Diagnostics, Shanghai, China).

\section{Statistical analysis}

The descriptive statistics for the continuous variables are expressed as median (quartiles), and the categorical variables are expressed as numbers (percentages). The continuous variables were compared using the Mann-Whitney test between 2 groups or Kruskal-Wallis test among 3 groups. The categorical variables were compared using the Chisquare test. Receiver operating characteristic (ROC) curves were plotted and the areas under the ROC curve (AUCs) were calculated to examine diagnostic efficiency. The Youden index was calculated to evaluate the diagnostic efficiency for each threshold. The threshold that maximized the Youden index was chosen as the optimal cutoff point.

To conduct the joint model for NSCLC diagnosis, patients with NSCLC or benign diseases that concurrently underwent CEA in combination with Cyfra21-1 and SCCA tests were included. To conduct the joint model for $\mathrm{ADC}$ diagnosis, patients with $\mathrm{ADC}$ or benign diseases that concurrently underwent CEA detection were included. To conduct the joint model for SCC diagnosis, patients with SCC or benign diseases that concurrently underwent both Cyfra21-1 and SCCA detection were included. To conduct the joint model for SCLC diagnosis, patients with SCLC or benign diseases that concurrently underwent a NSE test were included. The patients in each group were randomly assigned to the training set or validation set in 1:1 ratio, it was performed with 'R statistical software' using the 'caret' package (https://CRAN.R-project.org/package=caret). Different factors for malignant prediction and the joint diagnostic model were assessed by a binary logistic regression analysis.

The statistical analysis was performed using $\mathrm{R}$ and GraphPad prism8. All $\mathrm{P}$ values were based on 2-sided testing. A $\mathrm{P}$ value $<0.05$ was considered statistically significant.

\section{Results}

\section{Patients' characteristics}

Initially, 663 patients with suspicious pulmonary spaceoccupying been recruited by CT scanning, 32 were excluded for denying surgery or biopsy due to physical reasons, 16 were excluded because of acute respiratory tract infection (fungal, bacterial, viral or tuberculosis) in the past one month prior to enrolment, 36 patients who had been diagnosed with lung cancer and received anti-tumor therapy prior to enrolment, and 30 other malignancies were excluded, while 11 cases were confirmed calcified nodules were excluded as shown in Figure 1.

A total of 538 patients with suspicious pulmonary spaceoccupying lesions were recruited. Among the 282 (52.4\%) 


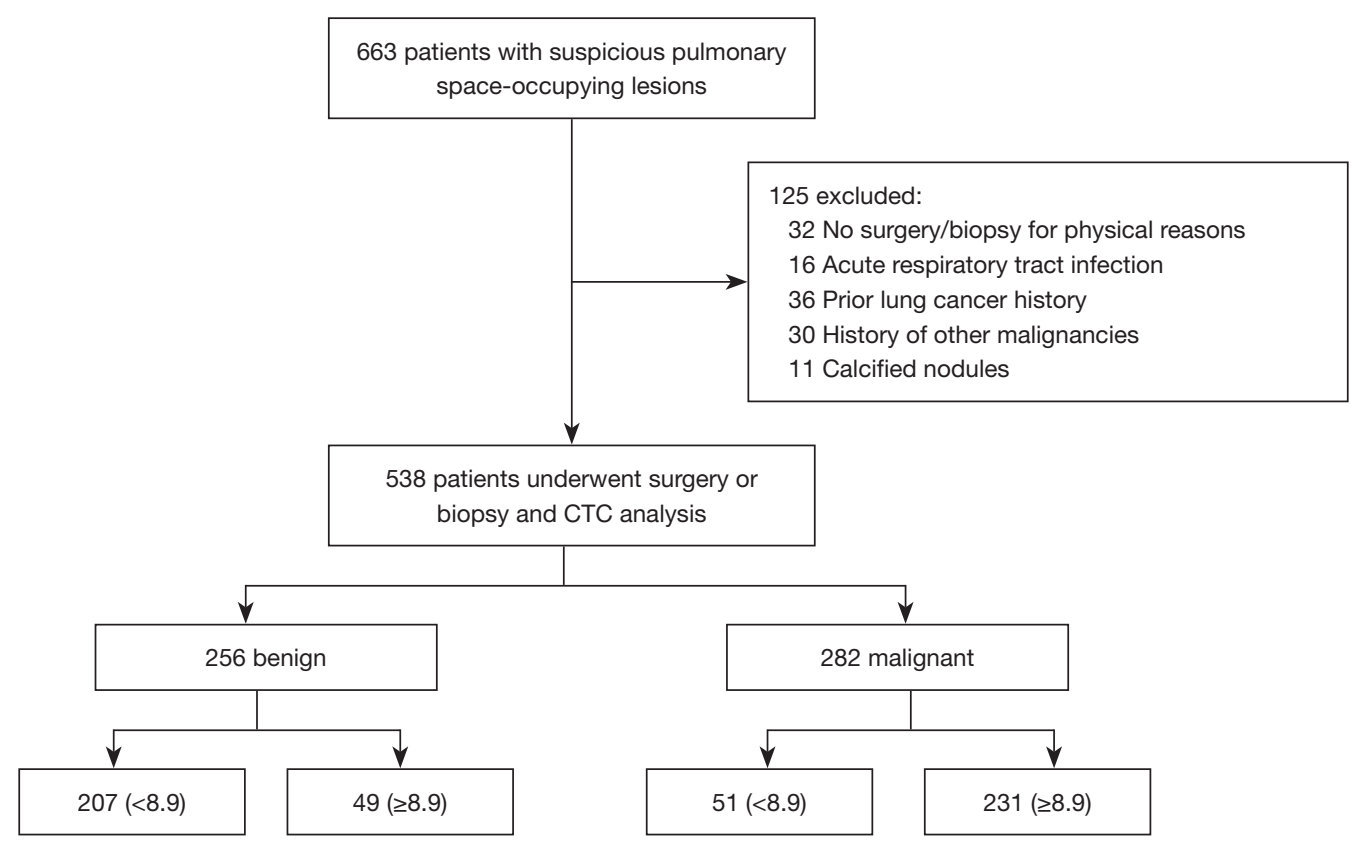

Figure 1 Flowchart of patient enrollment.

patients with malignant diseases, 187 were male and 95 were female, with an average age of $60.6 \pm 10.8$ years. Of these, $163(57.8 \%)$ were ADC cases, $71(25.2 \%)$ were SCC cases, and 48 (17\%) were small cell carcinoma cases. Among the 256 (47.6\%) patients with benign pulmonary space-occupying lesions, 129 were male and 127 were female, with an average age of $49.1 \pm 14.2$ years. Of these 35 (13.7\%) were granuloma cases, 22 (8.6\%) were hamartoma cases, $50(19.5 \%)$ were benign intrapulmonary nodule cases, 109 (42.6\%) were tuberculosis cases, $11(4.3 \%)$ were inflammatory pseudotumor cases, and $29(11.3 \%)$ were interstitial pulmonary disease cases.

There was no difference in the baseline characteristics of the training set and validation set in each subgroup. The characteristics of the patients in the training and validation sets are summarized in Table 1.

\section{FR+ CTC expression levels}

On the basis of the ROC curve, the optimal cutoff threshold for differentiating between patients with lung cancer and benign disease was $8.9 \mathrm{FU} / 3 \mathrm{~mL}$, with a sensitivity of $81.91 \%$ and specificity of $80.86 \%$ (Figure $2 A$ ). For patients in the malignant group, the median FR+ CTC level was $12.75(9.79,18.55) \mathrm{FU} / 3 \mathrm{~mL}$ with a range of $1.43-$ $40.8 \mathrm{FU} / 3 \mathrm{~mL}$ (Figure 2B). For patients in the benign group, the median FR+ CTC level was $6.65(4.93,8.30)$ $\mathrm{FU} / 3 \mathrm{~mL}$ with a range of $0.1-32.3 \mathrm{FU} / 3 \mathrm{~mL}$ (Figure $2 B$ ).

In relation to the pathological subtype classification of the malignant group, there was no significant difference in FR+ CTC levels among patients with ADC, SCC, and SCLC $(\mathrm{P}=0.3246$; Figure $2 C)$. We subsequently performed subgroup analyses on FR+ CTC levels in the benign group, and found a notable difference between patients with LD, Interstitial lung disease; IPT, inflammatory pseudotumor; GL, granuloma; $\mathrm{PH}$, pulmonary hamartoma; PN, pulmonary nodule; PTB, pulmonary tuberculosis $[6.75(5.28,7.90)$ vs. $7.24(5.50,12.63) v s$. $5.90(3.65,7.85) v s .6 .20(4.15,7.50)$ vs. $7.60(5.70,8.70)$ vs. $12.50(5.70,20.70) \mathrm{FU} / 3 \mathrm{~mL}, \mathrm{P}=0.0002$, respectively; Figure 2D].

\section{Diagnostic efficiency of FR+CTCs and classical serum tumor biomarkers}

To identify the best diagnostic biomarkers for different subgroups, ROC curves were plotted. The corresponding AUCs and $95 \%$ confidence intervals (CIs), sensitivity, specificity, positive predictive values (PPVs) and negative predictive values (NPVs) are summarized in Table 2. FR+ CTC displayed a higher AUC (training cohort, 0.865; 95\% CI: 0.808-0.923; validation cohort, 0.786 ; $95 \%$ CI: 
Table 1 Patient characteristics

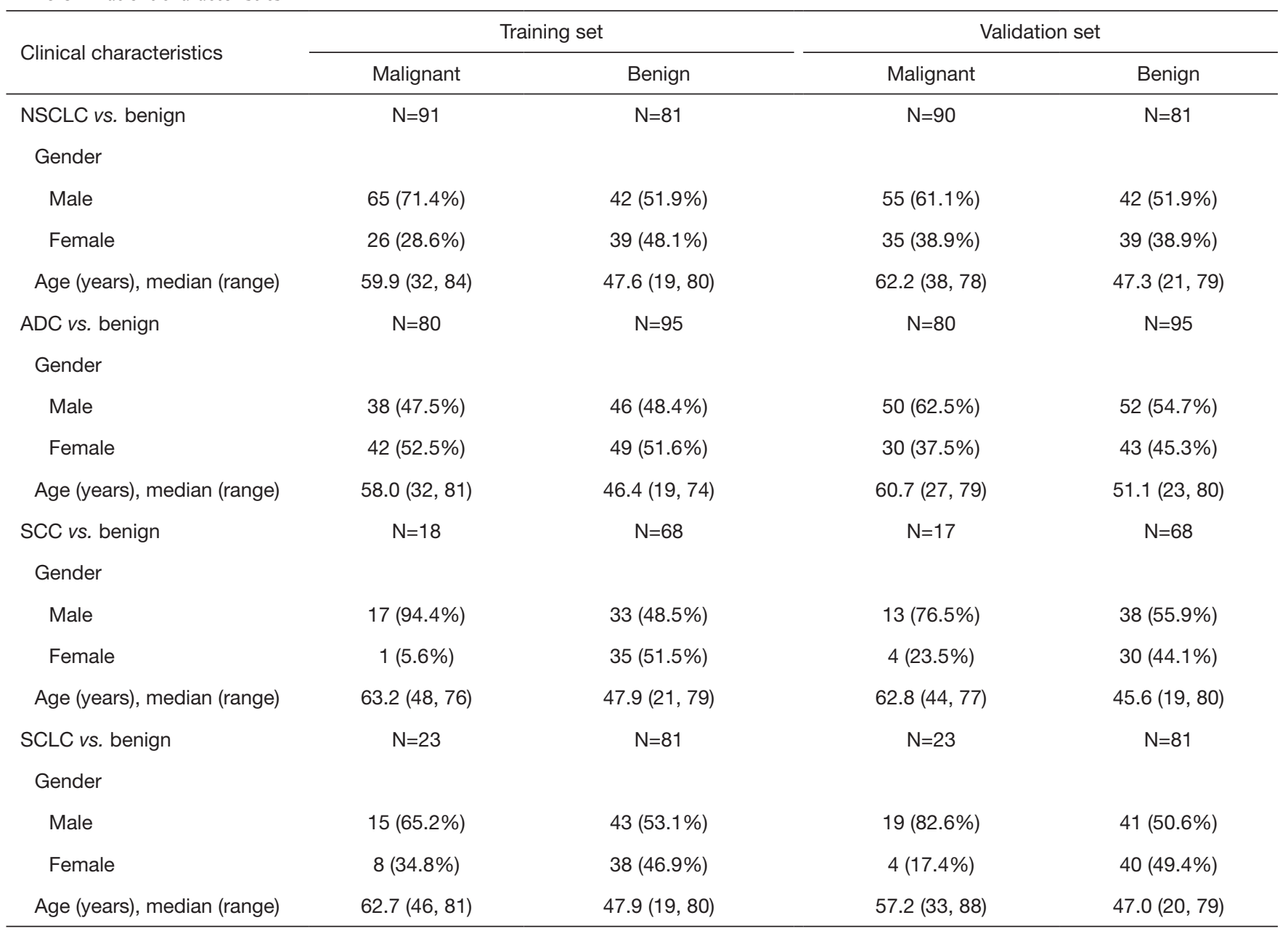

NSCLC, non-small cell lung cancer; ADC, adenocarcinoma; SCC, squamous cell carcinoma; SCLC, small cell lung cancer.

0.724-0.847; Table 2) than SCCA (training cohort, 0.496; 95\% CI: 0.402-0.590; validation cohort, 0.386; 95\% CI: 0.319-0.453; Table 2) and Cyfra21-1 (training cohort, 0.789; 95\% CI: 0.720-0.857; validation cohort, 0.765; 95\% CI: 0.704-0.827; Table 2) in differentiating between patients with NSCLC and benign space-occupying lung disease with sensitivity (training cohort, $80.2 \%$; validation cohort, $85.6 \%$ ) and specificity (training cohort, $88.9 \%$; validation cohort, 71.6\%). FR+ CTC (training cohort: AUC, 0.776; 95\% CI: 0.706-0.847; validation cohort: AUC, 0.823 ; 95\% CI: 0.767-0.878; Table 2) and CEA (training cohort: AUC, 0.852; 95\% CI: 0.796-0.909; validation cohort: AUC, 0.819; 95\% CI: 0.765-0.874; Table 2) displayed comparable efficiency in differentiating ADC and benign space-occupying lung disease. FR+ CTC had a higher AUC (training cohort, 0.828; 95\% CI: 0.699-0.957; validation cohort, 0.846; 95\% CI: 0.737-0.954; Table 2) than SCCA (training cohort, 0.731; 95\% CI: 0.556-0.907; validation cohort, 0.809; 95\% CI: 0.692-0.926; Table 2), and a lower AUC than Cyfra21-1 (training cohort, 0.904; 95\% CI: 0.828-0.981; validation cohort, 0.919; $95 \%$ CI: $0.851-$ 0.978; Table 2) in distinguishing between patients with SCC and benign space-occupying lung disease with a sensitivity (training cohort, $77.8 \%$; validation cohort, 76.5\%) and marked specificity (training cohort, $89.7 \%$; validation cohort, 92.6\%). Compared to NSE (training cohort: AUC, 0.836; 95\% CI: 0.721-0.951; validation cohort: AUC, 0.833; 95\% CI: 0.736-0.929; Table 2), FR+ CTC (training cohort: AUC, 0.851; 95\% CI: 0.756-0.945; validation cohort: AUC, 0.743; 95\% CI: 0.637-0.849; Table 2) displayed slightly lower efficiency in differentiating between patients with SCLC and lung benign space-occupying disease. 

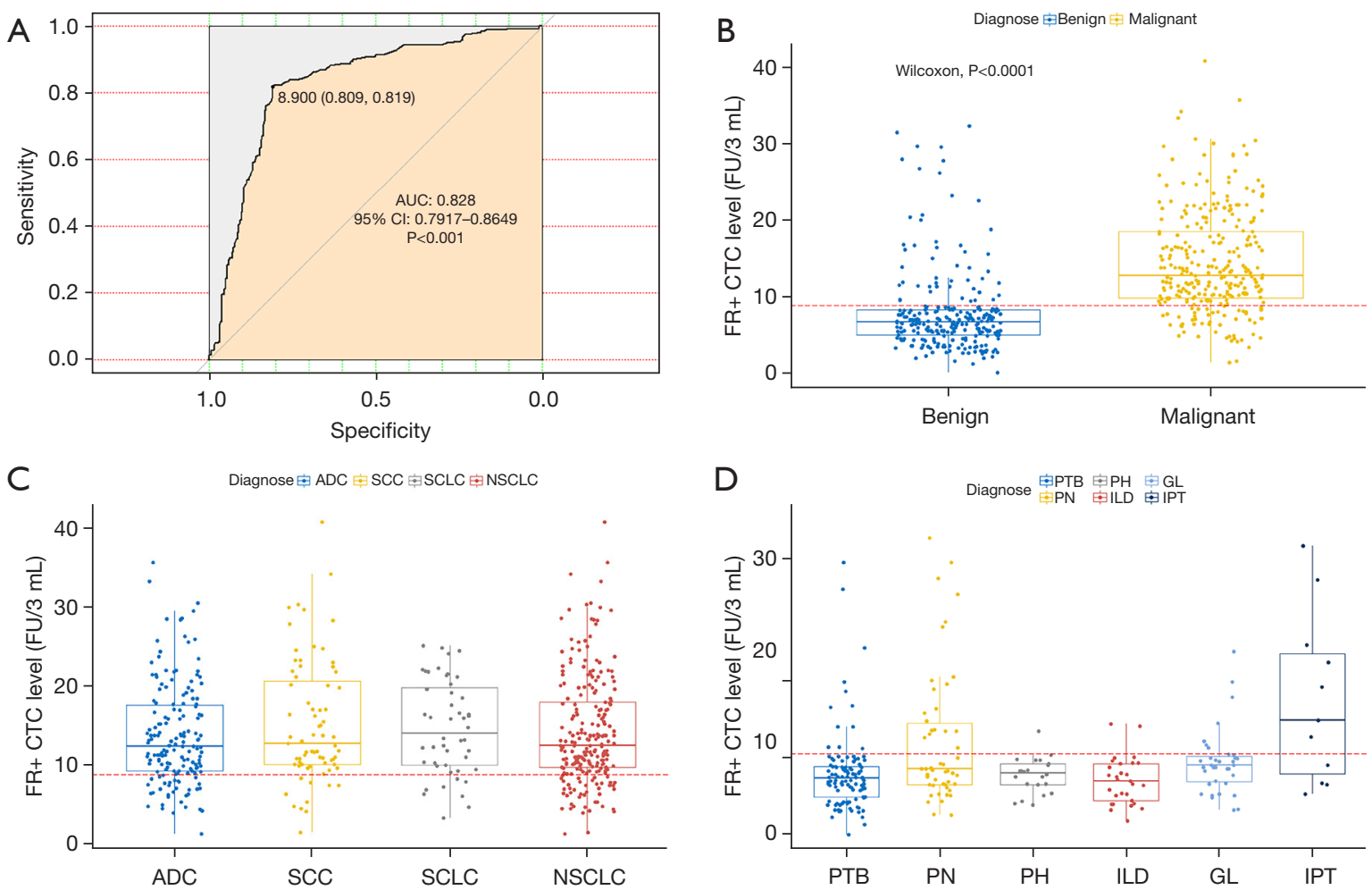

Figure 2 ROC analysis and comparison of FR+ CTC levels between subgroups. (A) ROC curves for diagnosis of lung cancer by FR+ CTCs; (B) the FR+ CTC levels in malignant vs. benign space-occupying lesions; (C) the FR+ CTC levels in different pathological subtypes of lung cancer; (D) the FR+ CTC levels in different benign respiratory diseases with space-occupying lesions. ROC, receiver operating characteristic; FR+ CTC, folate receptor-positive circulating tumor cells; ADC, adenocarcinoma; SCC, squamous cell carcinoma; SCLC, small cell lung cancer; NSCLC, non-small cell lung cancer.

Next, a logistic regression analysis of the combined lung cancer prediction model was performed. Based on the ROC curve analysis of the NSCLC subgroup, the Youden index was calculated and the cutoff values for FR+ CTC, CEA, Cyfra21-1, and SCCA were $8.9 \mathrm{FU} / 3 \mathrm{~mL}, 3.23 \mathrm{ng} / \mathrm{mL}$, $3.00 \mathrm{ng} / \mathrm{mL}$, and $0.75 \mathrm{ng} / \mathrm{mL}$, respectively. Patients with suspicious space-occupying pulmonary lesions were the dependent variables (benign space-occupying pulmonary lesions $=0$; malignant space-occupying pulmonary lesions =1), while FR+ CTCs, CEA, CYFRA21-1, and SCCA were the independent variables (negative $=0$, positive $=1$ ). Notably, the combination of FR+ CTC, SCCA, and Cyfra21-1 had better diagnostic efficacy at differentiating between patients with NSCLC and those with benign space-occupying lung disease than serum biomarkers alone. The AUCs of the joint model for differentiating between NSCLC and benign space-occupying lung disease were 0.968 (95\% CI: 0.944-0.993) in the training set, and 0.939 (95\% CI: 0.898-0.980) in the validation set (Figure 3A,3B,
Table 2). The AUCs of the joint model for ADC and benign space-occupying lung disease were 0.876 (95\% CI: $0.826-0.926)$ in the training set and 0.914 (95\% CI: $0.872-0.956$ ) in the validation set (Figure 3C,3D, Table 2). The AUCs of the joint model for SCC and benign spaceoccupying were 0.959 (95\% CI: 0.923-0.996) in the training set and 0.994 (95\% CI: $0.985-1.000)$ in the validation set (Figure 3E,3F, Table 2). The joint model that combined FR+ CTC and NSE had slightly better diagnostic efficacy for differentiating between SCLC and benign space-occupying lung disease than biomarkers alone (Figure 3G,3H, Table 2); however, the specificity (training set: $75.3 \%$; validation set: 74.1\%; Table 2) and PPV (training set: $53.5 \%$; validation set: $50.0 \%$; Table 2) of the joint model were dramatically decreased.

\section{Discussion}

Since the publication of the NLST study results in 1999, 
Table 2 The diagnostic efficiency of models in differentiating among patients with different types of lung cancer and benign space-occupying pulmonary diseases

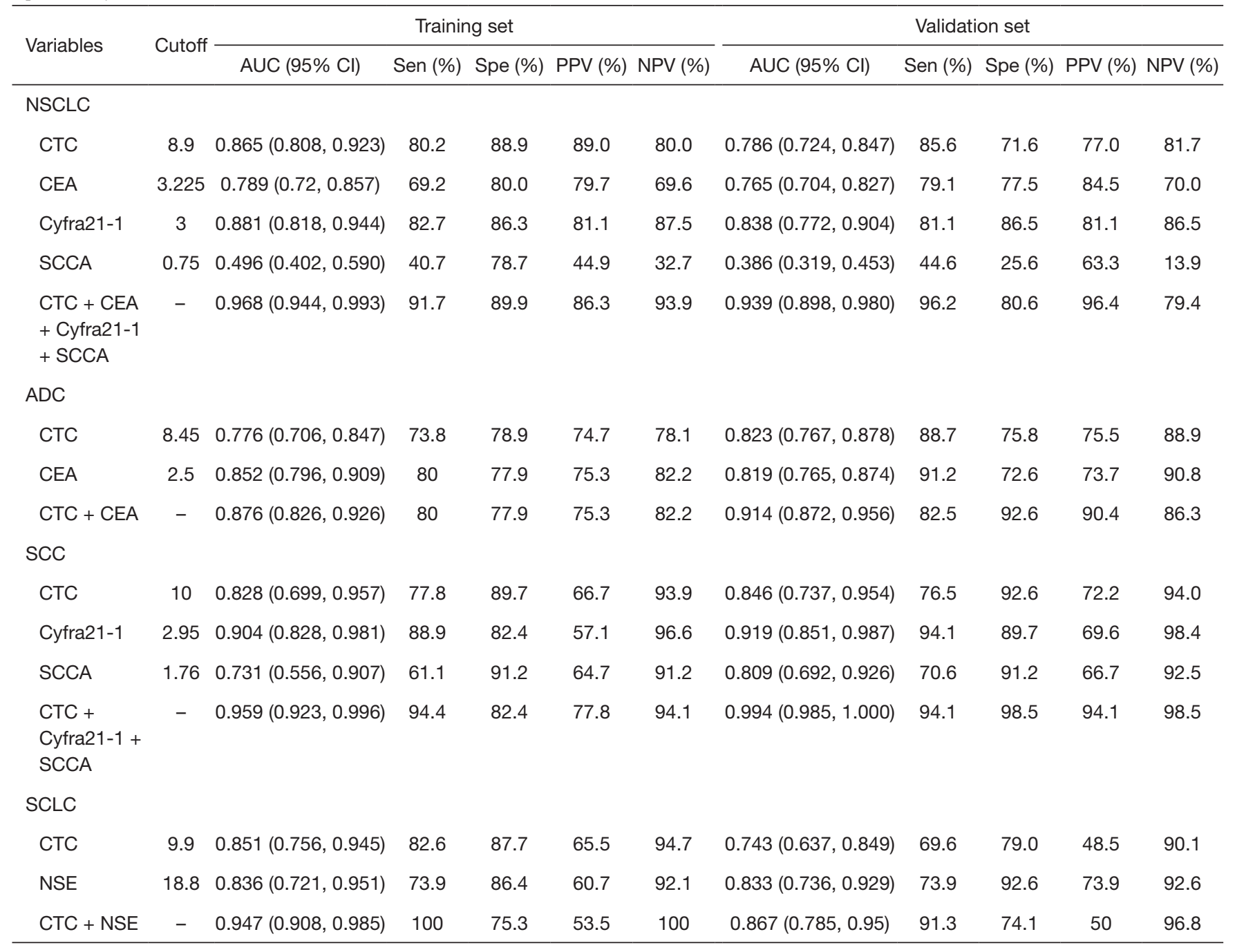

ADC, adenocarcinoma; CTC, circulating tumor cell; CEA, carcinoembryonic antigen; CYFRA21-1, cytokeratin 19 fragment; NSCLC, nonsmall cell lung cancer; NSE, neuron-specific enolase; PPV, positive predictive value; NPV, negative predictive value; SCC, squamous cell carcinoma; SCCA, squamous cell carcinoma antigen; SCLC, small cell lung cancer; Sen, sensitivity; Spe, specificity.

low-dose CT (LDCT) scans have been adopted by most clinical guidelines for regular lung cancer screenings of high-risk populations, but the definition of "high-risk" varies slightly among the guidelines (19). However, it has been reported that only $6.9-11.0 \%$ of space-occupying pulmonary lesions found by LDCT are malignant (20). Currently, the determination of malignancy is usually based on a combination of radiological features of the lesion, serum biomarkers, the patient's history, clinical symptoms, etc. Often, decisions to perform surgical resection/tissue biopsy or follow-up observations require a multidisciplinary team comprising pulmonologists, thoracic surgeons, radiologists, etc. More recently, a large number of institutions, including our own, have adopted "liquid biopsy" tests, many studies have reported that CTCs is an important potential biomarker for the diagnosis of various solid tumours (21-23), this requires that the detection method of CTCs must have high sensitivity and specificity. While folic acid receptor is a glycoprotein expressed on the surface of cell membrane, with strong tissue and tumor specificity, and is almost not expressed in blood cells and normal cells (except a small number of activated macrophages) of healthy subject (24), except in a few normal tissues, such as kidney, spleen and lung (25). FR 

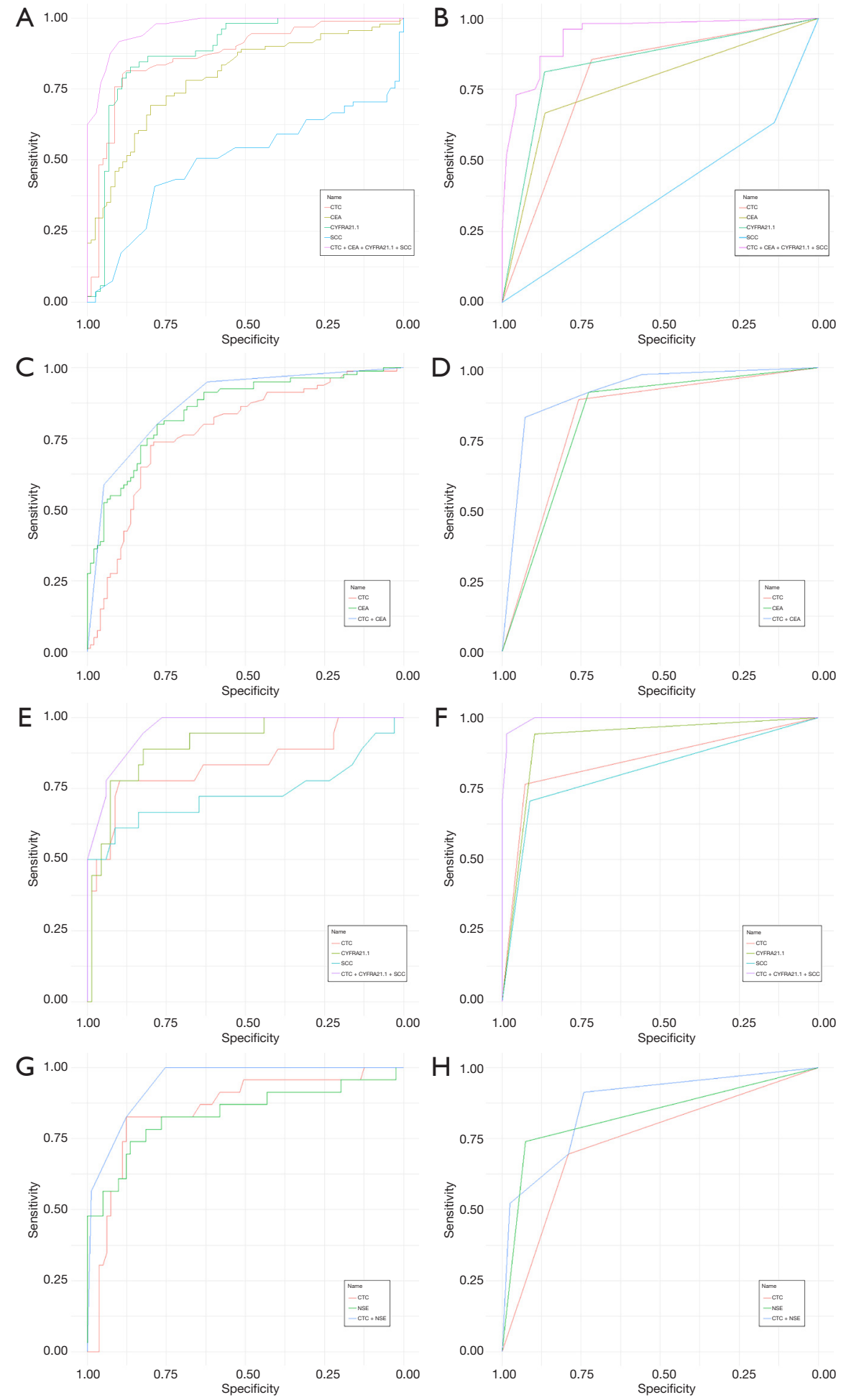

Figure 3 ROC curves of FR+ CTC in combination with serum biomarkers in pulmonary malignant tumors. (A,B) NSCLC vs. benign; (C,D) ADC vs. benign; (E,F) SCC vs. benign; (G,H) SCLC vs. benign. ADC, adenocarcinoma; FR+ CTC, folate receptor-positive circulating tumor cell; CEA, carcinoembryonic antigen; CYFRA21-1, cytokeratin 19 fragment; NSCLC, non-small cell lung cancer; NSE, neuronspecific enolase; ROC, receiver operating characteristic; SCC, squamous cell carcinoma; SCCA, squamous cell carcinoma antigen; SCLC, small cell lung cancer. 
can be an ideal target for CTCs detection. FR+ CTC test that was approved by the NMPA, to assist in the diagnosis of pulmonary lesions. Lou et. al. study the cut-off threshold between control group and initially diagnosed cancer group was 8.5 CTC unit, the sensitivity and specificity were $81.8 \%$, $93.2 \%$ (16). In Yu et. al. study the cutoff value between control groups and NSCLC group is 8.64 CTC units, with the sensitivity of $73.2 \%$, the specificity of $84.1 \%$ (17). Chen et. al. reported $\mathrm{FR}+\mathrm{CT}$ in differentiating patients with NSCLC from lung benign disease, with a remarkable sensitivity (72.46-76.37\%), and specificity $(82.39 \%$ $88.65 \%)$ in different cohort (15). In differentiating lung adenocarcinoma from lung benign disease, taking 8.35 units as the cutoff value for diagnosis, the diagnostic sensitivity was $70.2 \%$, with a specificity of $79.3 \%$ (26). Using 8.7 CTC Units $/ 3 \mathrm{~mL}$ as the cutoff threshold, the sensitivity and specificity of FR+ CTC were $81.94 \%$ and $73.08 \%$, respectively (18). With 8.3 CTC Units $/ 3 \mathrm{~mL}$ as the cutoff value, the sensitivity and specificity for differentiating malignant from benign nodules were $78.6-82.7 \%$ and $68.8-$ $78.4 \%$ (27). The FR+ CTC test has shown consistently high sensitivity and specificity in lung cancer diagnosis. In the present study, the sensitivity and specificity of FR+ CTC in diagnosing lung cancer was $81.9 \%$ and $80.9 \%$, respectively, which reflects the findings of previous studies (specificity: $82.4-93.2 \%$, sensitivity: $72.5-81.8 \%$ ) (14-18). And our results showed that FR+ CTC levels had the highest AUC compared to CEA and SCCA in the diagnosis of NSCLC, ADC, and SCC, and a comparable AUC compared to NSE in the diagnosis of SCLC. We found no difference in FR+ CTC levels among ADC, SCC, and SCLC patients, which also reflects the findings of previous studies (28). However, there is significant differences among lung benign space-occupying diseases, which may be due to the relatively higher FR+ CTC levels of pulmonary nodules $(40 \%)$ and inflammatory pseudotumors (63.6\%). Some benign pulmonary nodules are solely diagnosed on the clinical follow-up results, which may lead to false-positive results. We hypothesized that inflammatory diseases increased patients' white blood cell counts, which could lead to the insufficient depletion and the non-specific labelling of FR-specific probes to the remaining white blood cells after enrichment, and subsequently lead to false-positive results. Further, we compared the diagnostic yields of FR+ CTC with traditional serum tumor markers in our study. As we know, serum tumor biomarkers, such as CEA, SCC-Ag, Cyfra21-1, and NSE, are frequently used to help diagnose lung cancer in clinical practice. However, their sensitivity is still low, and false-positive results often occur due to infections, pregnancy, benign tumors, or other factors (29). Thus, FR+ CTC levels could serve as an effective auxiliary diagnostic marker for lung cancer.

We also assessed the diagnostic performance of the model via a multivariate evaluation. The diagnostic efficiency improved when FR+ CTCs were combined with serum makers in the diagnosis of NSCLC, ADC, SCC, and SCLC. Thus, the combined model should be recommended in clinical practice in the future. Notably, both the sensitivity and specificity of the NSCLC diagnostic model were higher than any biomarker alone, implying that the comprehensive analysis of FR+ CTC, CEA, Cyfra21-1, and SCCA provides a better approach for discriminating between benign space-occupying respiratory diseases and NSCLC. When FR+ CTC and NSE were combined in SCLC diagnosing, the sensitivity was $100 \%$ in the training group and $91.3 \%$ in the validation group, with a decrease in specificity to approximately $75 \%$, which suggests that other indicators, such as tumor size and age, need to be added to the diagnostic model to improve its specificity.

This preliminary study had several limitations. A portion of patients lacked pathological assessments. The diagnosis of some patients was confirmed based on their responses to related therapies (e.g., responses to antibiotics in tuberculosis); however, the diagnoses of some patients with pulmonary nodules were solely based on clinical observations, which may have led to discrepancies. Historically, 2-year follow ups of those determined to be non-malignant based on multidisciplinary evaluations indicate that over $90 \%$ do not progress to lung cancer (only considering the primary lesion; data not shown). Thus, the sole multidisciplinary evaluation of non-malignancy is relatively reliable.

To conclude, LT-PCR-based FR+ CTC detection can effectively distinguish between lung cancer and benign space-occupying respiratory diseases $(>8 \mathrm{~mm})$. It is worth mentioning that FR+ CTC levels could be used as a serviceable diagnostic biomarker for patients with NSCLC, especially in combination with common tumor markers. The test could also be useful in guiding the FR-based diagnostics and therapies in those patients with positive $\mathrm{FR}+\mathrm{CTC}$.

\section{Acknowledgments}

Funding: This study was supported by the Natural Science Basic Research Program of Shaanxi Province (No. 
2021JQ-352).

\section{Footnote}

Reporting Checklist: The authors have completed the STARD reporting checklist. Available at https://jtd.amegroups.com/ article/view/10.21037/jtd-21-1975/rc

Data Sharing Statement: Available at https://jtd.amegroups. com/article/view/10.21037/jtd-21-1975/dss

Conflicts of Interest: All authors have completed the ICMJE uniform disclosure form (available at https://jtd.amegroups. com/article/view/10.21037/jtd-21-1975/coif). The authors have no conflicts of interest to declare.

Ethical Statement: The authors are accountable for all aspects of the work in ensuring that questions related to the accuracy or integrity of any part of the work are appropriately investigated and resolved. The study was conducted in accordance with the Declaration of Helsinki (as revised in 2013). The ethics committee of the Xijing Hospital approved the study (No. KY20162056), and informed consent was obtained from each participant before study entry.

Open Access Statement: This is an Open Access article distributed in accordance with the Creative Commons Attribution-NonCommercial-NoDerivs 4.0 International License (CC BY-NC-ND 4.0), which permits the noncommercial replication and distribution of the article with the strict proviso that no changes or edits are made and the original work is properly cited (including links to both the formal publication through the relevant DOI and the license). See: https://creativecommons.org/licenses/by-nc-nd/4.0/.

\section{References}

1. Bhatt M, Kant S, Bhaskar R. Pulmonary tuberculosis as differential diagnosis of lung cancer. South Asian J Cancer 2012;1:36-42.

2. Winer-Muram HT. The solitary pulmonary nodule. Radiology 2006;239:34-49.

3. Gelbman BD, Cham MD, Kim W, et al. Radiographic and clinical characterization of false negative results from CTguided needle biopsies of lung nodules. J Thorac Oncol 2012;7:815-20.

4. Gould MK, Fletcher J, Iannettoni MD, et al. Evaluation of patients with pulmonary nodules: when is it lung cancer?: ACCP evidence-based clinical practice guidelines (2nd edition). Chest 2007;132:108S-30S.

5. Pastor A, Menéndez R, Cremades MJ, et al. Diagnostic value of SCC, CEA and CYFRA 21.1 in lung cancer: a Bayesian analysis. Eur Respir J 1997;10:603-9.

6. Tumour markers in lung cancer: EGTM recommendations. European Group on Tumour Markers. Anticancer Res 1999;19:2817-9.

7. Stieber P, Hatz R, Holdenrieder S, et al. National Academy of Clinical Biochemistry Guidelines for the Use of Tumor Markers in Lung Cancer. Tumour Biol 2006;27.

8. Peladan F, Monteil H. Gas chromatographic study of volatile fatty acids produced by 14 species of Pseudomonas. Ann Microbiol (Paris) 1984;135A:399-410.

9. Xu L, Su Z, Xie B. Diagnostic value of conventional tumor markers in young patients with pulmonary nodules. J Clin Lab Anal 2021;35:e23912.

10. Mateo J, Gerlinger M, Rodrigues DN, et al. The promise of circulating tumor cell analysis in cancer management. Genome Biol 2014;15:448.

11. O'Shannessy DJ, Gustavson M, Chandrasekaran LK, et al. Prognostic significance of FRA expression in epithelial cancers using AQUA(®) technology. Biomark Med 2013;7:933-46.

12. Iwakiri S, Sonobe M, Nagai S, et al. Expression status of folate receptor alpha is significantly correlated with prognosis in non-small-cell lung cancers. Ann Surg Oncol 2008;15:889-99.

13. Scaranti M, Cojocaru E, Banerjee S, et al. Exploiting the folate receptor $\alpha$ in oncology. Nat Rev Clin Oncol 2020;17:349-59.

14. Wang L, Wu C, Qiao L, et al. Clinical Significance of Folate Receptor-positive Circulating Tumor Cells Detected by Ligand-targeted Polymerase Chain Reaction in Lung Cancer. J Cancer 2017;8:104-10.

15. Chen X, Zhou F, Li X, et al. Folate Receptor-Positive Circulating Tumor Cell Detected by LT-PCR-Based Method as a Diagnostic Biomarker for Non-Small-Cell Lung Cancer. J Thorac Oncol 2015;10:1163-71.

16. Lou J, Ben S, Yang G, et al. Quantification of rare circulating tumor cells in non-small cell lung cancer by ligand-targeted PCR. PLoS One 2013;8:e80458.

17. Yu Y, Chen Z, Dong J, et al. Folate receptor-positive circulating tumor cells as a novel diagnostic biomarker in non-small cell lung cancer. Transl Oncol 2013;6:697-702.

18. Xue Y, Cong W, Xie S, et al. Folate-receptor-positive circulating tumor cells as an efficacious biomarker for the 
diagnosis of small pulmonary nodules. J Cancer Res Ther 2018;14:1620-6.

19. National Lung Screening Trial Research Team; Aberle DR, Adams AM, et al. Reduced lung-cancer mortality with low-dose computed tomographic screening. N Engl J Med 2011;365:395-409.

20. Pinsky PF, Gierada DS, Black W, et al. Performance of Lung-RADS in the National Lung Screening Trial: a retrospective assessment. Ann Intern Med 2015;162:485-91.

21. Tanaka F, Yoneda K, Kondo N, et al. Circulating tumor cell as a diagnostic marker in primary lung cancer. Clin Cancer Res 2009;15:6980-6.

22. Ilie M, Hofman V, Long-Mira E, et al. "Sentinel" circulating tumor cells allow early diagnosis of lung cancer in patients with chronic obstructive pulmonary disease. PLoS One 2014;9:e111597.

23. Maheswaran S, Sequist LV, Nagrath S, et al. Detection of mutations in EGFR in circulating lung-cancer cells. N Engl J Med 2008;359:366-77.

24. He W, Kularatne SA, Kalli KR, et al. Quantitation of circulating tumor cells in blood samples from ovarian and prostate cancer patients using tumor-specific fluorescent

Cite this article as: $\mathrm{Lv} \mathrm{X}, \mathrm{Wu} \mathrm{S}, \mathrm{Xu} X, \mathrm{Wu} \mathrm{L}$, Chang $\mathrm{N}$, Zhang J, Ti X. The combination of folate receptorpositive circulating tumor cells and serum tumor markers suggests a histological diagnosis of lung cancer. J Thorac Dis 2022;14(1):158-168. doi: 10.21037/jtd-21-1975 ligands. Int J Cancer 2008;123:1968-73.

25. Parker N, Turk MJ, Westrick E, et al. Folate receptor expression in carcinomas and normal tissues determined by a quantitative radioligand binding assay. Anal Biochem 2005;338:284-93.

26. Ding C, Zhou X, Xu C, et al. Circulating tumor cell levels and carcinoembryonic antigen: An improved diagnostic method for lung adenocarcinoma. Thorac Cancer 2018;9:1413-20.

27. Zhou Q, Geng Q, Wang L, et al. Value of folate receptor-positive circulating tumour cells in the clinical management of indeterminate lung nodules: A noninvasive biomarker for predicting malignancy and tumour invasiveness. EBioMedicine 2019;41:236-43.

28. Wan JW, Gao MZ, Hu RJ, et al. A preliminary study on the relationship between circulating tumor cells count and clinical features in patients with non-small cell lung cancer. Ann Transl Med 2015;3:352.

29. Jiang ZF, Wang M, Xu JL. Thymidine kinase 1 combined with CEA, CYFRA21-1 and NSE improved its diagnostic value for lung cancer. Life Sci 2018;194:1-6.

(English Language Editor: L. Huleatt) 